\title{
PGC-Enriched miRNAs Control Germ Cell Development
}

\author{
Jinhyuk Bhin ${ }^{1,5}$, Hoe-Su Jeong ${ }^{2,5}$ Jong Soo Kim², Jeong Oh Shin ${ }^{3}$, Ki Sung Hong', Han-Sung Jung ${ }^{3}$, \\ Changhoon Kim ${ }^{2}$, Daehee Hwang ${ }^{1,4 *}$, and Kye-Seong Kim ${ }^{2, *}$
}

\begin{abstract}
Non-coding microRNAs (miRNAs) regulate the translation of target messenger RNAs (mRNAs) involved in the growth and development of a variety of cells, including primordial germ cells (PGCs) which play an essential role in germ cell development. However, the target mRNAs and the regulatory networks influenced by miRNAs in PGCs remain unclear. Here, we demonstrate a novel miRNAs control PGC development through targeting mRNAs involved in various cellular pathways. We reveal the PGC-enriched expression patterns of nine miRNAs, including miR-10b, -18a, -93, 106b, -126-3p, -127, -181a, -181b, and -301, using miRNA expression analysis along with mRNA microarray analysis in PGCs, embryonic gonads, and postnatal testes. These miRNAs are highly expressed in PGCs, as demonstrated by Northern blotting, miRNA in situ hybridization assay, and miRNA qPCR analysis. This integrative study utilizing mRNA microarray analysis and miRNA target prediction demonstrates the regulatory networks through which these miRNAs regulate their potential target genes during PGC development. The elucidated networks of miRNAs disclose a coordinated molecular mechanism by which these miRNAs regulate distinct cellular pathways in PGCs that determine germ cell development.
\end{abstract}

\section{INTRODUCTION}

Primordial germ cells (PGCs) are the embryonic precursors of

${ }^{1}$ Department of Chemical Engineering, Pohang University of Science and Technology, Pohang 790-784, Korea, ${ }^{2}$ Graduate School of Biomedical Science and Engineering, Department of Biomedical Science, Hanyang University, Seoul 133-791, Korea, ${ }^{3}$ Division in Anatomy and Developmental Biology, Department of Oral Biology, Oral Science Research Center, BK21 PLUS Project, Yonsei University, College of Dentistry, Seoul 120-752, Korea, ${ }^{4}$ Department of New Biology and Center for Plant Aging Research, Institute for Basic Science, Daegu Gyeongbuk Institute of Science \& Technology, Daegu 711-873, Korea, ${ }^{5}$ These authors contributed equally to this work.

*Correspondence: ks66kim@hanyang.ac.kr (KKS); dhhwang@dgist.ac.kr $(\mathrm{DH})$

Received 26 May, 2015; revised 25 June, 2015; accepted 8 July, 2015; published online 7 October, 2015

Keywords: bioinformatic analysis, in situ hybridization, male primordial germ cells, microarray, miRNAs the gametes of the adult. During embryonic development, PGCs differentiate to germ cells, which develop oocytes in the female or spermatogonia in the male, and ultimately produce eggs or sperm, respectively. In mice, PGCs first emerge inside the extra-embryonic mesoderm at the posterior end of the primitive streak as a cluster of cells (Kucia et al., 2006) and then migrate toward the presumptive genital ridge, entering the genital ridge to colonize developing gonads. PGCs in gonads become distinguishable morphologically at 11.5 days post-coitum (dpc) and initiate differentiation toward the spermatogenic or oogenic morphogenesis at $12.5 \mathrm{dpc}$ (McLaren, 1992; 2000). They are regarded as the primordial pluripotent cells because of their unique ability to preserve the developmental totipotency (Abe, 2007; De Felici et al., 2009; McLaren, 2003). Embryonic germ cells derived from PGCs and embryonic stem cells (ESCs) from the inner cell mass (ICM) have many similarities, but PGCs and ICM exhibit distinct self-renewal capability and lineage-specific characteristics (Pal et al., 2009; Watson and Tam, 2001)

MicroRNAs (miRNAs) are 22 nucleotide, short, non-coding RNAs that regulate gene expression by inhibiting the transcription or translation of their target mRNAs (Bartel, 2004). Most miRNAs in animals function through the inhibition of effective mRNA translation of target genes through imperfect base pairing with the 3'-untranslated region (3' UTR) of target mRNAs. Thereby, the silencing mechanism of miRNAs is mediated by the inhibitory action of the RNA-induced silencing complex that both represses the translation and facilitates the degradation of targeted mRNAs (Filipowicz et al., 2008; Jaskiewicz and Filipowicz, 2008).

MiRNAs regulate growth and development in a variety of the cells (Kane et al., 2014). Also, a number of studies show that miRNAs regulate germ cell development (Banisch et al., 2012). During mouse germ cell development, the miR-290-295 and miR-17-92 clusters are known as significantly abundant miRNAs in PGCs. PGC-specific deficiency of Dicer, which plays a key role in microRNA biogenesis, results in poor PGC proliferation (Hayashi et al., 2008). MiR-290-295 cluster deficiency in mice results in embryonic lethality and germ cell defects with impaired PGC migration and premature ovarian failure (Medeiros et al., 2011). MiR-29b promotes female PGC development by targeting de novo DNA methyltransferase Dnmt3a and Dnmt3b (Takada et al., 2009). In chicken, miR-363 is involved in gonadal development (Huang et al., 2010). Moreover, miR-181a inhibits PGC differentiation by down-regulating 
HOXA1 and PGC meiosis by targeting NR6A1 (Lee et al., 2011). Although such studies demonstrate that miRNAs play an essential role in the regulation of gene expression during PGC development, the function of miRNAs and the regulatory network of their target mRNAs remain unclear.

The aim of the present study is to identify novel PGCenriched miRNAs that play an important role in PGC development and to reveal their regulatory networks. We performed a miRNA expression profiling analysis using a miRNA microarray and found nine novel miRNAs highly expressed in PGCs at $12.5 \mathrm{dpc}$ compared to gonocytes (GCs) at $15.5 \mathrm{dpc}$, spermatogonial stem cells (SSCs) at 5 days post-partum (dpp), testes at four and eight weeks as well as ESCs. To find the potential target genes whose expression may be in inverse proportion to PGC-enriched miRNA expression, we analyzed the messenger RNA expression profiling in PGCs at $12.5 \mathrm{dpc}$, SSCs at $5 \mathrm{dpp}$, and testes at $12 \mathrm{dpp}$ using an mRNA expression array. Therefore, we disclosed the potential target genes by PGC-enriched miRNAs and the regulatory networks of which PGC-enriched miRNAs regulate their target genes during PGC development. Collectively, PGC-enriched miRNAs determine germ cell development through regulating a variety of germ cell pathways, including cell growth, metabolic process, signaling, morphogenesis, and transcriptional regulation.

\section{MATERIALS AND METHODS}

\section{Sample preparation}

Fetal gonads were obtained from pregnant female ICR mice at 12.5 and $15.5 \mathrm{dpc}$ and testes were collected from male ICR mice at $5 \mathrm{dpp}, 12 \mathrm{dpp}$, four weeks, and eight weeks (Daehan Biolink Co. Ltd., Korea). Mouse R1 ES cell line (ATCC $®$ SCRC$1036^{\mathrm{TM}}$ ) was cultured with a medium supplemented with $15 \%$ fetal bovine serum, LIF, pyruvate, glutamine, $\beta$-mercaptoethanol, $100 \mathrm{U} / \mathrm{ml}$ penicillin, and $100 \mu \mathrm{g} / \mathrm{ml}$ streptomycin (Gibco BRL) in gelatin-coated plates and passaged with trypsin. The cells were cultured at $37^{\circ} \mathrm{C}$ in a humidified chamber containing $5 \% \mathrm{CO}_{2}$. All animal experiments were carried out in accordance with the National Institutes of Health guide for the care and use of laboratory animals (NIH Publications No. 8023, revised 1978). These animal studies were approved by the Institutional Animal Care and Use Committee of Hanyang University College of Medicine, Korea.

\section{miRNA microarray analysis}

Total RNAs were extracted from the cultured cells using Trizol reagent (Invitrogen, USA). For preparation of cellular miRNAs, small-sized RNAs containing miRNAs were isolated from total RNA using the RNeasy MinElute Cleanup kit (Qiagen, USA) according to the manufacturer's protocol. The isolated smallsized RNAs $(\sim 1 \mu \mathrm{g})$ were subjected to direct labeling with a fluorescent dye using the Platinum Bright 647 Infrared nucleic acid labeling kit (KREATECH, Netherland) according to the manufacturer's instruction. After labeling, labeled RNAs were purified from free fluorescent substrates using KREA pure columns (KREATECH, Netherland) according to the manufacturer's instruction, and used in hybridization. A hybridization assay was carried out with Genopal®-MICM miRNA arrays (Mitsubishi Rayon, Japan) overnight, where 180-oligonucleotide DNA probes are installed for detection of mouse miRNAs in $150 \mu \mathrm{l}$ of hybridization buffer containing $2 x \mathrm{SSC}, 0.2 \%$ SDS, and $\sim 1 \mu \mathrm{g}$ of heat-denatured labeled RNAs at $50^{\circ} \mathrm{C}$. After hybridization, the miRNA chips were washed twice in $2 x$ SSC containing $0.2 \%$ SDS at $50^{\circ} \mathrm{C}$ for 20 min followed by washing in $2 x$ SSC at $50^{\circ} \mathrm{C}$ for $10 \mathrm{~min}$. Hybridization signals were examined and analyzed using a DNA chip image analyzer according to the manufacturer's instruction (Mitsubishi Rayon, Japan).

\section{mRNA microarray analysis}

Total RNAs were amplified and purified using the Ambion Illumina RNA amplification kit (Ambion, USA) to yield biotinylated cRNA according to the manufacturer's instruction. Briefly, 550 ng of total RNAs was reverse-transcribed using an oligo (dT) primer and second-strand cRNA were synthesized and labeled with biotin-dNTP. After purification, cRNA were quantified using the ND-1000 Spectrophotometer (NanoDrop, USA). The 750 ng of labeled cRNA samples was hybridized to the Illumina MouseRef-8-v2-BeadChip overnight at $58^{\circ} \mathrm{C}$ according to the manufacturer's instruction (Illumina, Inc., USA). The array was scanned using the BeadStation 500 System (Illumina, USA) to acquire the signal of the probes. The data were normalized as log2 intensity values for each dataset using a quantile normalization method (Bolstad et al., 2003).

Reverse transcription-polymerase chain reaction (RT-PCR) One $\mu \mathrm{g}$ of DNase I-treatment total RNAs was reversetranscribed using Superscript II reverse transcriptase with random hexamer (Invitrogen) according to the manufacture's instruction. The standard PCR condition was as follows: 4 min at $94^{\circ} \mathrm{C}$, followed by $25-35$ cycles of $30 \mathrm{~s}$ at $94^{\circ} \mathrm{C}, 30 \mathrm{~s}$ at $55^{\circ} \mathrm{C}$, and $30 \mathrm{~s}$ at $72^{\circ} \mathrm{C}$. The primer sequences used in this study are given in Supplementary Table S1.

\section{miRNA quantitative real-time PCR assay}

TaqMan miRNA assay was performed as previously described (Chen et al., 2005). Reverse transcription (RT) reactions were run in a GeneAmp PCR 9,700 Thermocycler (Applied Biosystems, USA). RT reaction without templates or primer was used as the negative controls. Gene expression levels were quantified using the ABI 7300 RT-PCR System (Applied Biosytems). A comparative real-time PCR including no template control was performed in triplicate using each specific primer set for miR-93, miR-106b, miR-18a, miR-181a, miR-199a, miR-10b, mir-126, miR-181b, miR-301, miR-127, mir-210, mir-376a, and snoRNA234 as the loading control. The reaction was performed at $95^{\circ} \mathrm{C}$ for $5 \mathrm{~min}$, followed by 40 cycles of $95^{\circ} \mathrm{C}$ for $15 \mathrm{~s}$, and $60^{\circ} \mathrm{C}$ for $1 \mathrm{~min}$. All reagents and protocols were from Applied Biosystems (USA). The expression level of each miRNA relative to snoRNA234 was determined using the $2^{-\Delta \Delta C t}$ method (Livak and Schmittgen, 2001).

\section{Northern blot analysis}

The total RNAs were isolated from the cultured cells, including R1 ESCs, Sertoli cells, and MEF cells, and ICR mouse tissue (male gonads at $12.5 \mathrm{dpc}$ and $15.5 \mathrm{dpc}$, testes at $5 \mathrm{dpp}$, four weeks, and eight weeks) using a Trizol reagent (Invitrogen, USA). Northern blotting was performed as previously described (Lagos-Quintana et al., 2001). The total RNA were separated in a $12.5 \%$ polyacrylamide gel containing $7 \mathrm{M}$ urea, transferred to a Hybond-N+ membrane (GE Healthcare Life Sciences), and fixed by an ultraviolet cross-linker. Probes were end-labeled with $[\gamma-32 P]$ ATP (GE Healthcare Life Sciences) using T4 polynucleotide kinase (Takara, Japan). Prehybridization, hybridization, and wash were carried out at $42^{\circ} \mathrm{C}$ using ExpressHyb Hybridization Solution (Clontech, USA) according to the manufacturer's instruction. Blots were exposed on BioMax MS film (Kodak) at $-80^{\circ} \mathrm{C}$ for $48 \mathrm{~h}$. The $18 \mathrm{~S}$ and $28 \mathrm{~S}$ ribosomal RNA bands were stained with ethidium bromide and used as a loading 
control. The sequences of probes were as follows: mmu-miR-93; 5'-caaagtgctgttcgtgcaggt ag-3', mmu-miR-106a; 5'-caa agtgctaacagtgcaggt ag- $3^{\prime}$, mmu-miR-181b; $5^{\prime}$-aacatt cat tgctgtcgg tgg gt-3', mmu-miR-18; 5'-taaggtgcatctagtgca gat ag-3'.

\section{miRNA in situ hybridization assay}

For miRNA in situ hybridization, PGCs at $12.5 \mathrm{dpc}$ and testes at 5 and 12 dpp were fixed with $4 \%$ paraformaldehyde (PFA) in 0.01 MPBS ( $\mathrm{pH} 7.4$ ) overnight at $4^{\circ} \mathrm{C}$. After embedding in paraffin, the tissue was sectioned in $7 \mathrm{~mm}$ thicknesses. The sections were then baked at $65^{\circ} \mathrm{C}$, de-waxed in xylene, rehydrated through graded washes of ethanol in water, and post-fixed in $4 \%$ PFA. The sections were pre-warmed and hybridized with the 5'-digoxigenin labeled locked nucleic acid (LNA) microRNA probes in a humid chamber containing $50 \%$ formamide in $2 x$ SSC overnight at $20-25^{\circ} \mathrm{C}$ (Kloosterman et al., 2006). After washing with $2 x$ SSC, the sections, an anti-DIG-antibody in TBS containing $10 \%$ FBS was applied and incubated at $4^{\circ} \mathrm{C}$ overnight. After washing with TBS, the sections were incubated in NTMT, and color development was enhanced by NBT and BCIP. The probes of miR-106b, $-93,-18 a$, and $-181 a$, and scramble were obtained from Exiqon.

\section{Bioinformatics}

Identification of potential target genes by miRNAs: Potential targets by PGC-enriched miRNAs were generated through three miRNAs-target prediction programs, including TargetScan (Lewis et al., 2005), mirDB (Wang, 2008), and mirBase (GriffithsJones et al., 2006), in regard to the genes of each mRNA expression profiles. Mouse transcripts predicted to be miRNA targets were retrieved from the Sanger microRNA database.

Identification of differentially expressed genes (DEGs); We first defined the expressed genes across ICR mouse tissues at $12.5 \mathrm{dpc}, 5 \mathrm{dpp}$, and $13.5 \mathrm{dpp}$ using a Gaussian mixture modeling method as previously described (Lee et al., 2010). Among potential candidates, DEGs were identified by following three comparisons: 1) $12.5 \mathrm{dpc}$ versus $5 \mathrm{dpp}$, 2) $12.5 \mathrm{dpc}$ versus 12 dpp, 3) $5 \mathrm{dpp}$ versus $12 \mathrm{dpp}$ using an integrative statistical method previously reported (Chae et al., 2013). This method calculated adjusted $P$-values $(P)$ by combining $P$-values from two-sample $t$-test and $\log _{2}$-median ratio test using empirical distributions of T-values and $\log _{2}$-median ratios by random permutations between samples. For each comparison, the DEGs were defined as the genes with $\mathrm{P}<0.05$ and log2-fold-changes $>0.58$. DEGs were grouped into the eighteen clusters based on their differential expression patterns over time (Supplementary Fig. S1).

Gene ontology analysis: Gene ontology enrichment analysis was performed using a DAVID software (Huang da et al., 2009). Gene ontology Biological Processes (GOBPs) with P-value < 0.1 (a default cut-off) computed from DAVID were selected as the ones enriched by the genes in each group.

\section{Statistical analysis}

Unless stated otherwise, each experiment was carried out using at least three replicates. Data were described as mean \pm SEM. Statistically significant differences between groups were evaluated by one-way analysis of variance (ANOVA) using a log-linear model in the Statistical Analysis System (SAS, USA). Values of $\mathrm{P}<0.05$ were considered statistically significant.

\section{RESULTS}

PGC-enriched miRNAs during germ cell development
To examine the novel miRNAs predominantly expressed in PGCs during germ cell development, we performed miRNA expression profiling arrays in PGCs at $12.5 \mathrm{dpc}$, gonads at 15.5 $\mathrm{dpc}$, testes at four and eight weeks, ESCs, Sertoli cells, and MEF cells using the Genopal®-MICM miRNA arrays as described in "Materials and Methods". Among the 180 miRNAs, we selected 12 miRNAs that were expressed specifically in PGCs with criteria of a significant $(P<0.05)$ increase $(>1.5-$ fold) in PGCs compared to controls. These 12 mRNAs, including miR-10b, -18a, -93, -106b, -126-3p, -127, -181a, -181b, -301, $-199 a,-210$, and $-376 a$, were expressed highly in PGCs at 12.5 $\mathrm{dpc}$ and gonads at $15.5 \mathrm{dpc}$ compared to other controls (Fig. 1A). The expression levels of these miRNAs decreased with mature germ cell development (Fig. 1A). Of the 12 miRNAs, some miRNAs, such as miR-301, $-106 \mathrm{~b},-93$, and $-18 a$, were expressed highly in PGCs, whereas miR-199a, $-127,-376 a$, and -210 were expressed more in gonads at $15.5 \mathrm{dpc}$. Therefore, 12 miRNAs meet the criteria as potential regulatory microRNA candidates that are highly and specifically expressed in PGCs.

The accuracy of the miRNA microarray analysis is affected by the short length of mature miRNA sequences leading to an increase in the false discovery rate. Therefore, we conducted miRNA qPCR analysis to validate the PGC-enriched miRNA expression (Fig. 1B). Based on the restriction criteria of miRNA expression in PGCs, we isolated nine PGC-enriched miRNAs including miR-10b, -18a, -93, -106b, -126-3p, -127, -181a, -181b, and -301 , during PGC development. miR-199a, -210, and -376a were also expressed in PGCs (Supplementary Fig. S2), but the expression level of these miRNAs was smaller compared to that of the selected nine miRNAs (data not shown). Since the PGC-enriched expression of miR-199a, -210, and -376a fell below selection criteria, these miRNAs were excluded from PGC-enriched miRNAs.

A Northern blot analysis confirmed again the PGC-enriched miRNA expression of miR-18a, -93, -106b, and -181a (Fig. 1C). To narrow our conclusion, we conducted a miRNA in situ hybridization assay to examine the PGC-enriched miRNA expression of miR-18a, $-93,-106 \mathrm{~b}$, and $-181 \mathrm{a}$. These miRNAs were expressed precisely in PGCs at $12.5 \mathrm{dpc}$ (Fig. 1D), but not in other tissues and other germ stages (Supplementary Fig. S3). Together, our data indicate that nine novel miRNAs are expressed in a PGC-dependent fashion, suggestive of their roles in regulating $P G C$ development.

\section{Target genes by PGC-enriched miRNAs}

Our results indicate that $P G C$-enriched miRNAs may regulate PGC development. To further establish this, we performed an integrative analysis using the comparison of the mRNA expression profiling between PGCs at $12.5 \mathrm{dpc}$, SSCs at $5 \mathrm{dpp}$, and testes at $12 \mathrm{dpp}$. We found a total of 4,171 DEGs from three comparisons as follows: 1) $12.5 \mathrm{dpc}$ versus $5 \mathrm{dpp}$ (2,313 DEGs), 2) $12.5 \mathrm{dpc}$ versus $12 \mathrm{dpp}$ (2,177 DEGs), and 3) $5 \mathrm{dpp}$ versus $12 \mathrm{dpp}$ (2,226 DEGs). These DEGs were assembled into the eighteen expression patterns (C1-C18) (Supplementary Fig. S1A and Supplementary Table S2). Among them, the gene expression patterns in $\mathrm{C} 1$ (29 genes) and C2 (343 genes) were inversely correlated with the PGCenriched miRNA expression (Fig. 2A and Supplementary Fig. $\mathrm{S} 1 \mathrm{~B})$. It seems that some genes in $\mathrm{C} 1$ and $\mathrm{C} 2$ may be the potential targets by PGC-enriched miRNAs. To test the notion, we checked the potential targets of our nine miRNAs using three microRNA target prediction programs; TargetScan, mirDB, and mirBase (Griffiths-Jones et al., 2006; Lewis et al., 2005; Wang, 2008). According to our analysis, a total of 6,511 


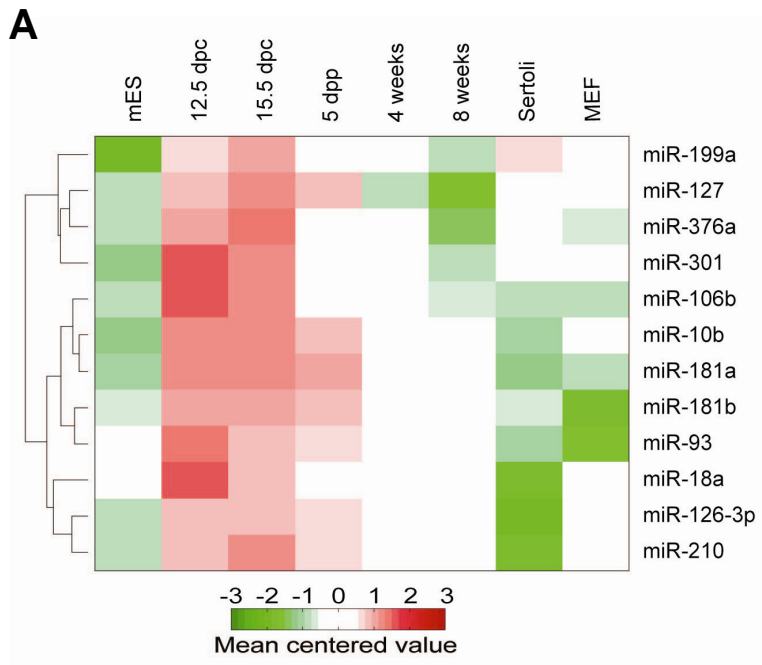

C

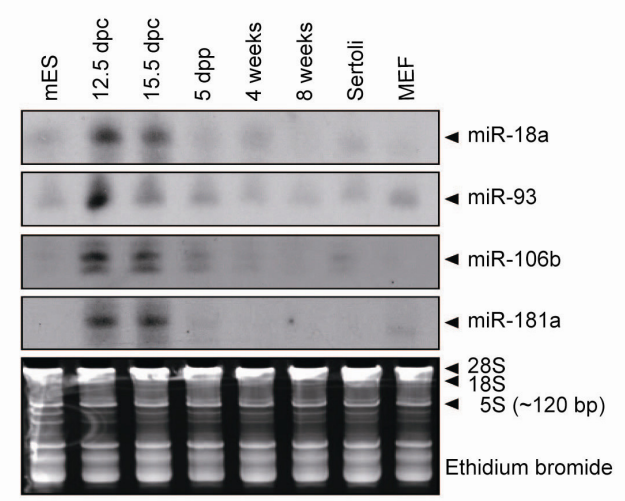

B

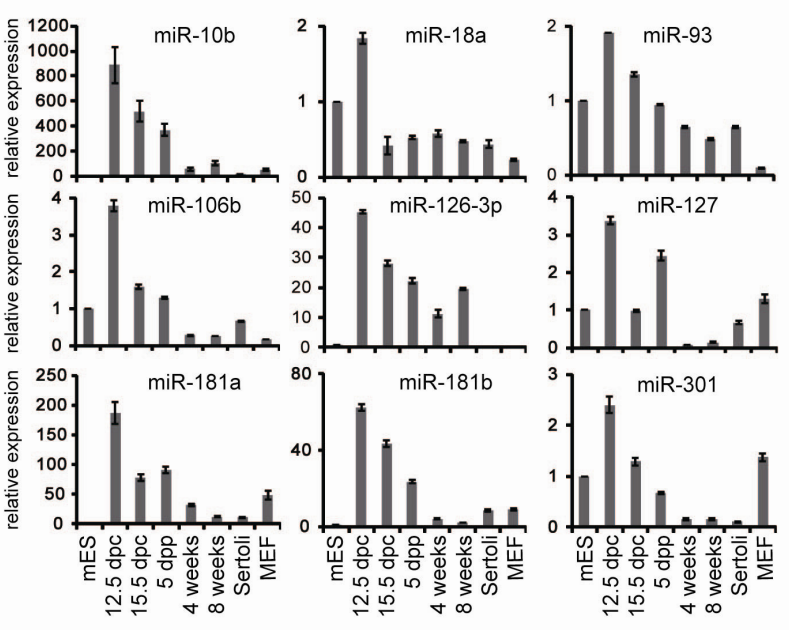

$\boldsymbol{D}$

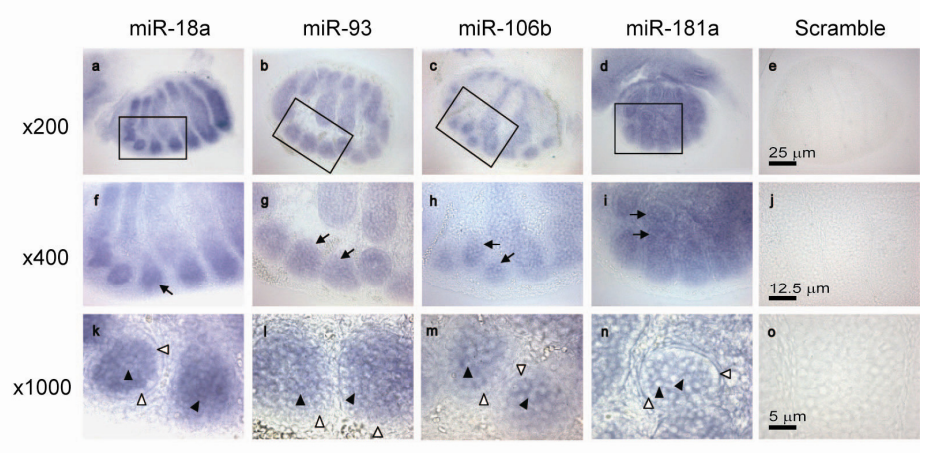

Fig. 1. The expression levels of miRNAs during male germ cell development. (A) Expression profiling of 12 PGC-enriched miRNAs identified by miRNA microarray in developing male germ cells. For each miRNA, the mean-centered $\log _{2}$-expression profile $(m e a n=0)$ is shown. $A$ hierarchical clustering was done to visualize the similarity in expression patterns of the miRNAs. The colors represent increased (red) and decreased (green) expression relative to the mean expression level. (B) PGC-enriched expression of miR-10b, -18a, -93, -106b, -126-3p, $-127,-181 \mathrm{a},-181 \mathrm{~b}$, and -301 (red) among 12 miRNAs identified from a miRNA microarray analysis were confirmed by miRNA qPCR. (C) Northern blot analysis demonstrates that miR-18a, -93, -106b, and -181a were expressed in PGCs during male germ cell development. In the bottom, Ethidium bromide staining of $28 \mathrm{~S}, 18 \mathrm{~S}$, and $5 \mathrm{~S}$ rRNAs is shown as a loading control. Northern blot assay was performed as described in Materials and Methods. (D) miRNA in situ hybridization assay demonstrated the PGC-enriched expression patterns of miRNAs in PGCs at $12.5 \mathrm{dpc}$. Arrow, a seminiferous cord; black arrowhead, primordial germ cells; white arrowhead, Sertoli cells.

genes was targeted by miR-10b, -18a, -93, -106b, -126-3p, 127, $-181 \mathrm{a},-181 \mathrm{~b}$, and -301 . The 127 genes could be the potential targets because of affiliating to $\mathrm{C} 1$ and $\mathrm{C} 2$ (Fig. 2B and Supplementary Table S3). As expected, the expression pattern of 127 genes exhibited a reverse relationship with that of PGC-enriched miRNAs (Fig. 2C). Interestingly, miR$106 \mathrm{~b}$ and -93 are reported to be transcribed together as a common polycistronic transcript and to be a member of the same miRNA cluster, miR-17-92 cluster (Olive et al., 2010). Thus, miR-106b and -93 have highly similar mature sequences and may share target genes. In line with that possibility, miR-106b and -93 shared 39 common targets among a total of 49 genes (Fig. 2D and Supplementary Table S3). Additionally, miR-181a and $-181 \mathrm{~b}$ are clustered on chromosome 1 and co-transcribed. MiR-181a and $-181 \mathrm{~b}$ had 28 common targets among a total of 36 potential genes (Fig. 2D and Supplementary Table S3).

\section{PGC-enriched miRNAs regulate cellular processes}

PGCs arrived at the genital ridges and become incorporated into sex cords at $11.5 \mathrm{dpc}$. They continued to proliferate until 13.5-14.5 dpc and then entered a quiescent period. After birth, GCs resumed mitosis and initiated their differentiation into spermatogonia. During this process, GCs undergo a variety of possible morphological and metabolic changes including apoptosis (Fujimoto et al., 2000; Honke et al., 2002; Zhang et al., 2005). To explore the physiological effects on GC development by stage specific expression of nine candidate miRNAs, we examined the cellular processes represented by 127 target genes based on the enrichment analysis of GOBPs using DAVID software (Huang da et al., 2009). The GOBPs of the 127 target genes indicated their involvement in germ cell development mechanisms, such as cell replication, morphogenesis, apoptosis, regulatory intracellular signaling cascades, and phospholipid metabolic processes (Fig. 3A and Supplementary 
$\boldsymbol{A}$

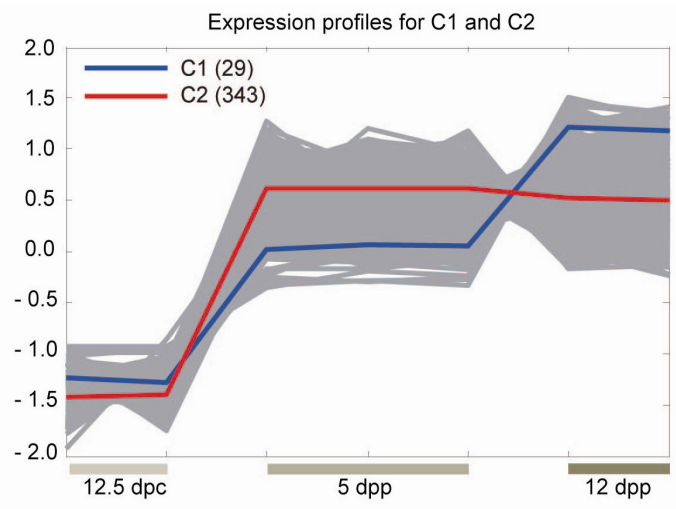

C

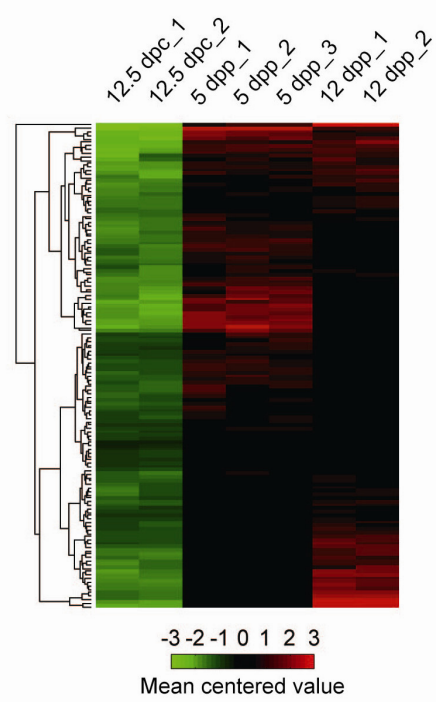

B

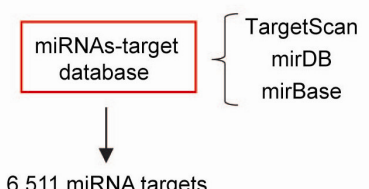

(miR-10b, -18a, -93, -106b, -126-3p, -127, -181a, -181b, and -301)

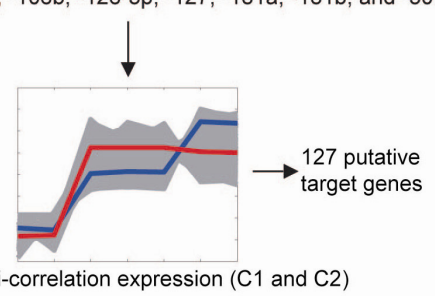

D

\begin{tabular}{|c|c|c|c|c|}
\hline \multicolumn{4}{|l|}{ niR-181a } & miR-181b \\
\hline Al316807 & 1110012J17Rik & Gdpd1 & Rasl10a & \\
\hline $\mathrm{Ccl} 7$ & Arhgef3 & $\mathrm{H} 2-\mathrm{K} 1$ & Reep1 & Rnf19a \\
\hline Col7a1 & Camta2 & Luzp1 & $\operatorname{Rin} 2$ & \\
\hline Fam92a & Celf2 & Magea5 & Sgpp1 & \\
\hline Oat & Clic6 & Man2a1 & Slc19a2 & \\
\hline Osgin1 & Cpne2 & Mbnl1 & Snrk & \\
\hline \multirow[t]{4}{*}{ Rnaset $2 \mathrm{~b}$} & Cyo5b & Mtap1b & Trak1 & \\
\hline & Dazap2 & $\mathrm{Nacc2}$ & Uыз & \\
\hline & Egr1 & Pnrc2 & & \\
\hline & Gas7 & Prika & & \\
\hline
\end{tabular}

Fig. 2. Identification of target genes by PGC-enriched miRNAs and their biological processes. (A) The expression of target genes categorized in $\mathrm{C} 1$ and $\mathrm{C} 2$ showed a reverse correlation with nine PGC-enriched miRNAs during germ cell development. The auto-scaled log $_{2}$-expression profiles of each target gene (mean $=0$ and standard deviation $=1$ ) are shown (gray line). Median expression values in $\mathrm{C} 1$ and $\mathrm{C} 2$ for each sample are expressed as blue and red lines. (B) The strategy for identifying target genes by nine PGC-enriched miRNAs. 6,511 targets were generated from the three databases using the sequence pairing algorithms. Among them, 127 were selected as target genes having a reverse expression ( $\mathrm{C} 1$ and $\mathrm{C} 2$ ) with miRNA expression. (C) A heat map showed expression patterns of the 127 target genes. For each gene, the mean-centered $\log _{2}$-expression profile $($ mean $=0$ ) is shown. A hierarchical clustering was done to visualize the similarity in expression patterns of the genes. The colors represent increased (red) and decreased (green) expression relative to the mean expression level. (D) miR-106b and -93 shared target genes due to possession of similar seed sequence. Similarly, miR-181a and $-181 \mathrm{~b}$ shared target genes.

Table S4). Our results suggest that the miRNA-specific targets could be down-regulated after birth to prevent precocious germ cell development. Similarly, the induction of target genes coinciding with regulation of cell cycle and apoptosis after birth suggests support for the resumption of mitosis and the restriction of germ cell loss necessary for proper germ cell development as previously noted (Kondoh et al., 1991).

Regulatory networks affected by PGC-enriched miRNAs MiRNAs are known to regulate germ cell development (Banisch et al., 2012). To understand the regulatory roles of miRNAs in PGC development, we demonstrated a network of which nine miRNAs appeared to regulate PGC development through changing target gene expression. Of the 127 target genes as members of $\mathrm{C} 1$ and $\mathrm{C} 2,92$ genes were clustered to the known functions based on their GOBP annotations according to DAVID software (Huang da et al., 2009). Thus, we created a network model containing the 12 major modules grouped by the 92 nodes (Fig. 3B), including 1) apoptosis, 2) cell cycle and proliferation, 3) differentiation and morphogenesis, 4) structure organization, 5) intracellular signaling cascades, 6) transcription regulation, 7) transport and localization, 8) cell adhesion and migration, 9) cellular metabolic process, 10) RNA processing, 11) proteolysis, and 12) immune and defense response.

To explore the information-theoretic models for complex networks, we assembled the relative contribution by each PGCenriched miRNAs in response to the 12 cellular processes (Fig. $4 A)$. We calculated the score as a relative contribution of target 
miRNA Has Important Role in PGC Development Jinhyuk Bhin et al.

$\boldsymbol{A}$

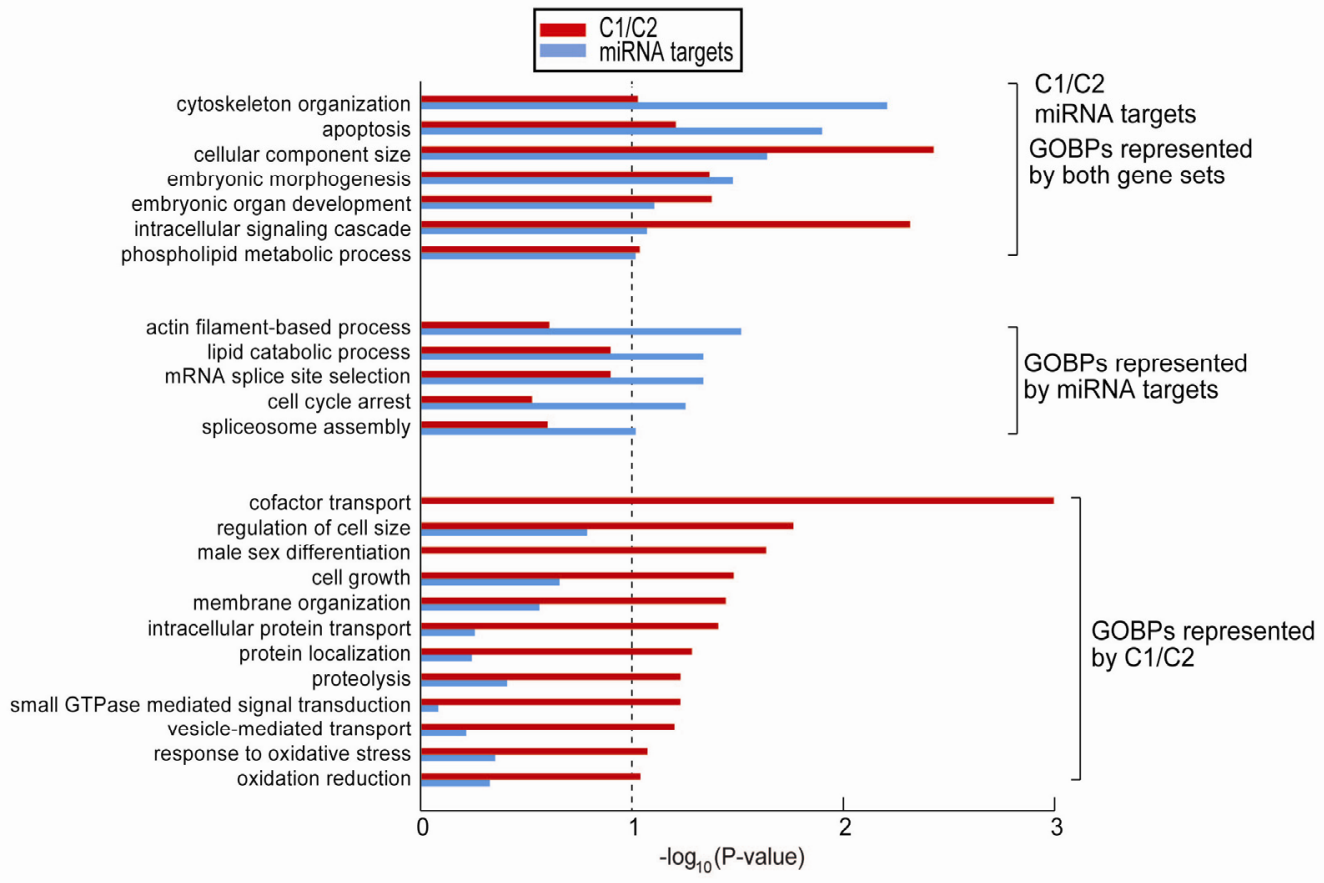

B

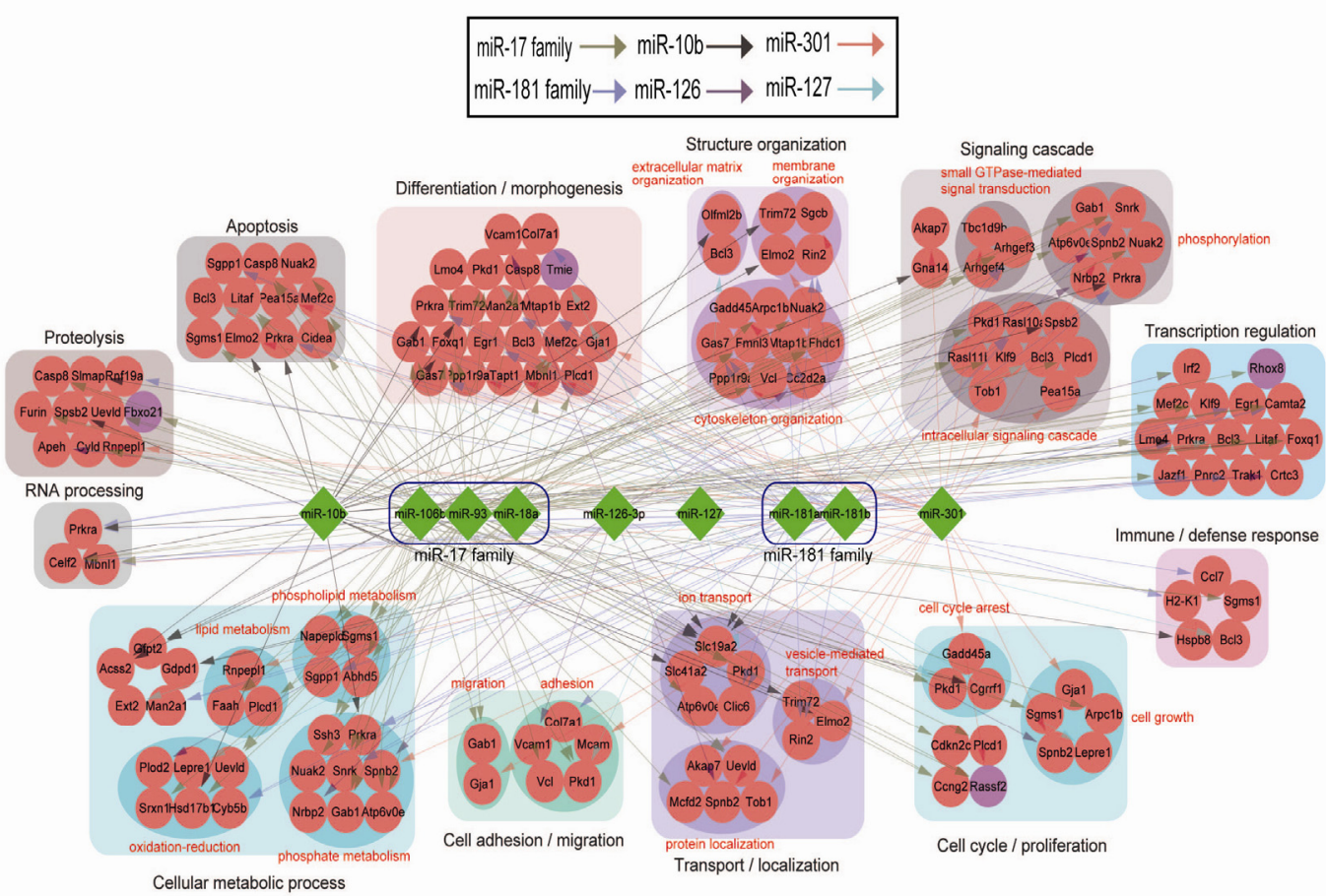

Fig. 3. A network model delineates biological processes regulated by PGC-enriched miRNAs. (A) The enrichment analysis results of GOBPs by total genes in $\mathrm{C} 1$ and $\mathrm{C} 2$ (red bar, total genes) and 127 target genes (blue bar). The red line denotes the cutoff value $(P$-value $=0.1)$ of the analysis. (B) A network model shows the relationships between nine PGC-enriched miRNAs and their target genes. Colors and shapes of the nodes represent PGC-enriched miRNAs (green diamonds), target genes in C1 (purple circles) and C2 (red circles). Edge colors were used to distinguish the interactions of individual miRNAs or miRNA families. The nodes were grouped according to their gene ontologies (colored rectangles). Nodes containing the same gene ontologies belong to same group as described in "Materials and Methods". The genes with multiple gene ontologies were comprised of two or more functional groups. The background colors represent gene ontology groups (ellipsoidal for lower-level gene ontologies and rectangle for upper-level gene ontologies). 
$\boldsymbol{A}$

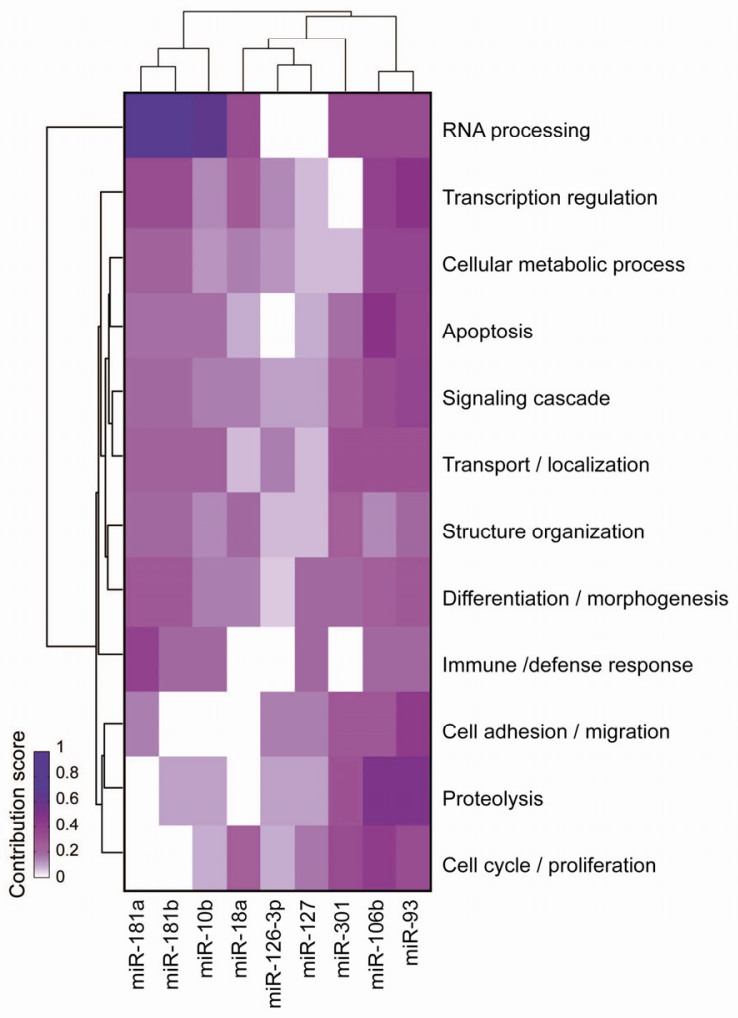

B

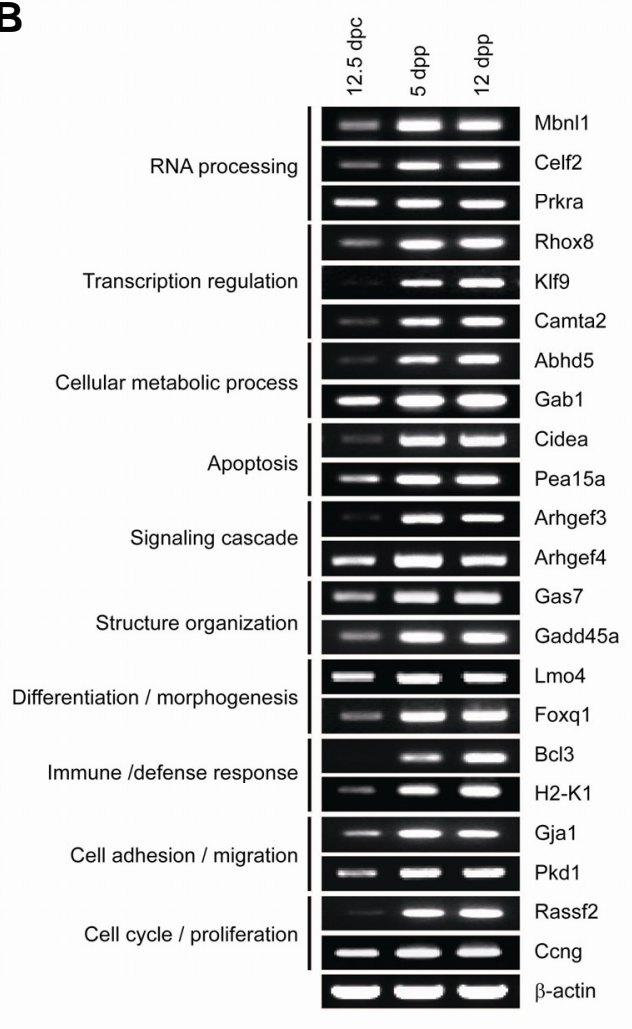

Fig. 4. A heat map represents the relationship between each PGC-enriched miRNA and the twelve biological processes. (A) A heat map shows the contribution score defined by the number of target genes regulated by each miRNA within a gene ontology group divided by the total number of the target genes of the miRNA within the group. (B) RT-PCR analysis confirms expression of the representative target genes during male germ cell development.

genes by individual miRNAs. Notably, this analysis revealed that the miR-17-92 cluster, including miR-93 and $-106 \mathrm{~b}$, plays a crucial role in the regulation of the 12 cellular processes during germ cell development. Especially, the contribution score implied that miR-93 and $-106 \mathrm{~b}$ have an anti-apoptotic role by targeting apoptotic Cidea (Figs. 3A and 4B, and Supplementary Table S3). Our prediction is consistent with the previous conclusions that miR-106b acts as a hub molecule in an apoptotic miRNA-gene network (Liu et al., 2012) and the suppression of miR-106b-93-25 cluster expression is essential for ER stressmediated apoptosis (Gupta et al., 2012). To corroborate the above suggested correlations in light of a network model, the effect on expression levels of target genes by individual miRNAs was examined by RT-PCR (Fig. 4B). As target gene expression was indeed decreased with germ cell development, it is suggestive that these targets might be regulated by our miRNAs.

Moreover, the contribution score implicated that miR-106b and -93 have a role in specific metabolic processes, especially phospholipid metabolism, through targeting Abhd5 and Gab1 (Fig. 4B). Moreover, miR-18a, another member of miR17 family, regulated a broad spectrum of processes for germ cell development, such as differentiation/morphogenesis, RNA processing, transcription regulation, and cell cycle, by targeting Rhox8, Klf9, Camta2, Mbnl1, Celf2, Lmo4, Foxq1, Rassf2, and Ccng2 (Fig. 4B). Our suggestion is in agree- ment with the previous observation that miR-18a regulates male germ cell maturation (Bjork et al., 2010). Furthermore, miR-181a and $-181 \mathrm{~b}$ negatively regulated the expression of targets, including Mbnl1 and Prkra, that modulate differentiation and development based on the contribution score (Fig. 4B). Therefore, miR-10b, -18a, -181b, and -301 along with miR-181a, which is expressed highly in chicken PGCs, might cooperatively regulate germ cell development, repress PGC differentiation, and keep PGCs from entering meiosis. Finally, the contribution analysis showed that other PGC-enriched miRNAs, such as miR-10b, -126-3p, -127, and -301 , may regulate diverse cellular processes during germ cell development. Based on the contribution score, 1) miR-126-3p could regulate cell adhesion/migration, transport/localization, and transcription regulation, 2) miR-10b may regulate apoptosis, RNA processing, transport/localization, and immune/defense response, 3) miR-127 might regulate differentiation/morphogenesis, and 4) miR-301 could regulate cell cycle/proliferation, structure organization, signaling cascade, differentiation/morphogenesis, and adhesion/migration. Taken together, our results demonstrate that nine PGCenriched miRNAs play a key role in the regulation of a variety of cellular processes related to PGC development. Furthermore, our network model could extend the current understanding of the regulatory roles of miRNAs during germ cell development. 


\section{DISCUSSION}

MiRNAs play an essential role in regulation of specification, migration, and differentiation in the gametes (Dyce et al., 2010; Jessberger, 2008; Tang, 2010).Though the Surani group reported that miRNAs, such as miR-290-295 and miR-17-92 clusters, are expressed highly in PGCs along with germ cell development (Hayashiet al., 2008), a systematic analysis of PGC-enriched miRNAs has not been explored. In this study, we find that nine novel miRNAs, including miR-10b, -18a, -93, $-106 b,-126-3 p,-127,-181 a,-181 b$, and -301 , are expressed selectively in PGCs according to our comparative study profiling miRNA expression of PGCs at $12.5 \mathrm{dpc}$, GCs at $15.5 \mathrm{dpc}$, SSCs at $5 \mathrm{dpp}$, and testes at four and eight weeks. To understand the functional roles of these miRNAs, we investigated the potential target genes by an integrative analysis with the sequence pairing information and anti-correlated expression profile data between miRNAs and target mRNAs. Using the antirelationship between miRNAs and their target genes, we are able to present a network model delineating how these nine miRNAs regulate the PGC developmental processes, including differentiation and morphogenesis, cell proliferation and apoptosis, cell adhesion and migration, cellular signaling cascades, and other cellular metabolic processes.

A variety of miRNA target prediction algorithms are based on the sequence pairing between miRNAs and target transcripts (Griffiths-Jones et al., 2006; Lewis et al., 2005). Recently, the miRNA integrative analysis using in silico predictions and the data of microRNA and mRNA expression profiling shows an inverse expression correlation between miRNA and target mRNA. This method provides more precise target prediction compared to the conventional sequence pairing algorithms (Huang et al., 2007; Joung et al., 2007; Peng et al., 2009; Tran et al., 2008; Wang, 2008). Using a newly developed integrative analysis, we propose a network model to understand the regulatory roles of nine PGC-enriched miRNAs.

Of the nine miRNAs, miR-18a, -93 , and $-106 b$ belonging to the miR-17-92 cluster were previously reported to be highly expressed in mouse PGCs (Hayashi et al., 2008). In addition, miR-181a was known to be highly expressed in chicken PGCs and to inhibit the somatic differentiation of PGCs by silencing homeobox A1 (Lee et al., 2011). On the other hand, five miRNAs, such as miR-10b, -126-3p, -127, $-181 b$, and -301 , have not been previously reported in relation to PGC development. miR-18a, one of the nine PGC-enriched miRNAs, may mediate male germ cell maturation by inhibiting a transcription factor HSF2 that influences a wide range of developmental process (Bjorket al., 2010). Although we do not find that HSF2 belongs to our DEGs in PGCs development, our network model suggests that some target genes, such as Camta2, Mbnl1, Rhox8, and Foxq1, targeted by miR-18a may contribute to PGC development. Two clusters of the miR-17 family, miR-17-92 and miR106b-25 clusters, might regulate germ cell differentiation by decreasing Bim, Kit, Socs3, and Stat3 (Tong et al., 2012). Moreover, Gab1, Casp8, Foxq1, Lmo4, Pkd1, and Mef2c by miR-106b and/or -93 possibly will control PGC development. Vcam-1, which is targeted by the miR-126-3p (Harris et al., 2008) and Pkd1, which is targeted by the miR-127 along with miR -93 and $-106 \mathrm{~b}$, mediate cell adhesion/migration and differentiation/morphology, suggesting that miR-126-3p and -127 may be involved with cell adhesion/migration and differentiation/morphology in PGCs. Additionally, the miR-181 family including miR-181a and -181b expresses in PGCs of the chicken (Lee et al., 2011), as well as in human (Sirotkin et al., 2009) and mouse (Saunders et al., 2010). Sirtuin-1 is a target of miR-181a (Zhou et al., 2012). Sirtuin-1 null mice showed to be sterile in males and sirtuin-1 also participated with growth hormone/insulin-like growth axis (Xu et al., 2010). Collectively, the miR-181 family including miR-181a and -181 b play a key role in reproductive development.

During germ cell development, massive changes in gene control and expression, such as alternative splicing and mitoticto-meiotic transition also occur (Schmid et al., 2013). In support of these, the miR-181a/b and miR-10b modulate mechanisms of RNA processing, such as spliceosome assembly and mRNA splicing site selection in PGCs (Fig. 4A and Supplementary Tables S3). Moreover, Tbc1d9b, which is targeted by miR-301, and Arhgef3 and Arhgef4, whose expression is decreased by the miR-17 family, mediate small GTPase-mediated signaling pathways, suggesting miR-17 family and miR-301 involvement in Ras signaling in PGCs.

In summary, our study provides a demonstration and comprehensive bioinformatics analysis of nine novel miRNAs that are likely candidates to be important in PGC miRNA-mediated regulatory networks involved in development of PGCs, such as those regulating essential mechanisms of differentiation and morphogenesis, cell proliferation and apoptosis, cell adhesion and migration, and cellular signaling cascades, as well as other cellular metabolic processes.

Note: Supplementary information is available on the Molecules and Cells website (www.molcells.org).

\section{ACKNOWLEDGMENTS}

We are grateful to Professor Eric B. Richardson, M.D., Hanyang University, for giving the constructive comments and editing our manuscript. This work was supported by grant from the Medical Research Center (2008-0062190) and by Institute of Basic Science (IBS-R013-G1-2015) funded by the National Research Foundation of Korea (NRF) of the Ministry of Science, ICT \& Future Planning, Republic of Korea.

\section{REFERENCES}

Abe, K. (2007). Developmental program for pluripotential cells and primordial germ cells in mice. Tanpakushitsu Kakusan Koso 52, 2046-2053.

Banisch, T.U., Goudarzi, M., and Raz, E. (2012). Small RNAs in germ cell development. Curr. Topics Dev. Biol. 99, 79-113.

Bartel, D.P. (2004). MicroRNAs: genomics, biogenesis, mechanism, and function. Cell 116, 281-297.

Bjork, J.K., Sandqvist, A., Elsing, A.N., Kotaja, N., and Sistonen, L. (2010). miR-18, a member of Oncomir-1, targets heat shock transcription factor 2 in spermatogenesis. Development 137, 3177-3184

Bolstad, B.M., Irizarry, R.A., Astrand, M., and Speed, T.P. (2003). A comparison of normalization methods for high density oligonucleotide array data based on variance and bias. Bioinformatics 19, 185-193.

Chae, S., Ahn, B.Y., Byun, K., Cho, Y.M., Yu, M.H., Lee, B., Hwang, D., and Park, K.S. (2013). A systems approach for decoding mitochondrial retrograde signaling pathways. Sci. Signal. 6, rs4.

Chen, C., Ridzon, D.A., Broomer, A.J., Zhou, Z., Lee, D.H., Nguyen, J.T., Barbisin, M., Xu, N.L., Mahuvakar, V.R., Andersen, M.R., et al. (2005). Real-time quantification of microRNAs by stem-loop RT-PCR. Nucleic Acids Res. 33, e179.

De Felici, M., Farini, D., and Dolci, S. (2009). In or out stemness: comparing growth factor signalling in mouse embryonic stem cells and primordial germ cells. Curr. Stem Cell Res. Ther. 4, 8797.

Dyce, P.W., Toms, D., and Li, J. (2010). Stem cells and germ cells: microRNA and gene expression signatures. Histol. Histopathol. 25, 505-513. 
Filipowicz, W., Bhattacharyya, S.N., and Sonenberg, N. (2008). Mechanisms of post-transcriptional regulation by microRNAs: are the answers in sight? Nat. Rev. Genet. 9, 102-114.

Fujimoto, H., Tadano-Aritomi, K., Tokumasu, A., Ito, K., Hikita, T., Suzuki, K., and Ishizuka, I. (2000). Requirement of seminolipid in spermatogenesis revealed by UDP-galactose: Ceramide galactosyltransferase-deficient mice. J. Biol. Chem. 275, 2262322626.

Griffiths-Jones, S., Grocock, R.J., van Dongen, S., Bateman, A. and Enright, A.J. (2006). miRBase: microRNA sequences, targets and gene nomenclature. Nucleic Acids Res. 34, D140-144.

Gupta, S., Read, D.E., Deepti, A., Cawley, K., Gupta, A., Oommen, D., Verfaillie, T., Matus, S., Smith, M.A., Mott, J.L., et al. (2012). Perk-dependent repression of miR-106b-25 cluster is required for ER stress-induced apoptosis. Cell Death Dis. 3, e333.

Harris, T.A., Yamakuchi, M., Ferlito, M., Mendell, J.T., and Lowenstein, C.J. (2008). MicroRNA-126 regulates endothelial expression of vascular cell adhesion molecule 1. Proc. Natl. Acad. Sci. USA 105, 1516-1521.

Hayashi, K., Chuva de Sousa Lopes, S.M., Kaneda, M., Tang, F., Hajkova, P., Lao, K., O'Carroll, D., Das, P.P., Tarakhovsky, A., Miska, E.A., et al. (2008). MicroRNA biogenesis is required for mouse primordial germ cell development and spermatogenesis. PLoS One 3, e1738.

Honke, K., Hirahara, Y., Dupree, J., Suzuki, K., Popko, B., Fukushima, K., Fukushima, J., Nagasawa, T., Yoshida, N., Wada, Y., et al. (2002). Paranodal junction formation and spermatogenesis require sulfoglycolipids. Proc. Natl. Acad. Sci. USA 99, 4227-4232.

Huang, J.C., Babak, T., Corson, T.W., Chua, G., Khan, S., Gallie, B.L., Hughes, T.R., Blencowe, B.J., Frey, B.J., and Morris, Q.D. (2007). Using expression profiling data to identify human microRNA targets. Nat. Methods 4, 1045-1049.

Huang, P., Gong, Y., Peng, X., Li, S., Yang, Y., and Feng, Y. (2010). Cloning, identification, and expression analysis at the stage of gonadal sex differentiation of chicken miR-363 and $363^{*}$. Acta Biochim. Biophys. Sin (Shanghai) 42, 522-529

Huang da, W., Sherman, B.T., and Lempicki, R.A. (2009). Systematic and integrative analysis of large gene lists using DAVID bioinformatics resources. Nat. Protocols 4, 44-57.

Jaskiewicz, L., and Filipowicz, W. (2008). Role of Dicer in posttranscriptional RNA silencing. Curr. Top Microbiol. Immunol. 320, 77-97.

Jessberger, R. (2008). New insights into germ cell tumor formation. Horm. Metab. Res. 40, 342-346.

Joung, J.G., Hwang, K.B., Nam, J.W., Kim, S.J., and Zhang, B.T. (2007). Discovery of microRNA-mRNA modules via populationbased probabilistic learning. Bioinformatics 23, 1141-1147.

Kane, N.M., Thrasher, A.J., Angelini, G.D., and Emanueli, C. (2014). Concise review: MicroRNAs as modulators of stem cells and angiogenesis. Stem Cells 32, 1059-1066.

Kloosterman, W.P., Wienholds, E., de Bruijn, E., Kauppinen, S., and Plasterk, R.H. (2006). In situ detection of miRNAs in animal embryos using LNA-modified oligonucleotide probes. Nat. Methods 3, 27-29.

Kondoh, G., Murata, Y., Aozasa, K., Yutsudo, M., and Hakura, A. (1991). Very high incidence of germ cell tumorigenesis (seminomagenesis) in human papillomavirus type 16 transgenic mice. J. Virol. 65, 3335-3339.

Kucia, M., Machalinski, B., and Ratajczak, M.Z. (2006). The developmental deposition of epiblast/germ cell-line derived cells in various organs as a hypothetical explanation of stem cell plasticity? Acta Neurobiol. Exp. (Wars) 66, 331-341.

Lagos-Quintana, M., Rauhur, R., Lendeckel, W., and Tuschl, T. (2001). Identification of novel genes coding for small expressed RNAs. Science 294, 853-858.

Lee, H.J., Suk, J.E., Patrick, C., Bae, E.J., Cho, J.H., Rho, S. Hwang, D., Masliah, E., and Lee, S.J. (2010). Direct transfer of alpha-synuclein from neuron to astroglia causes inflammatory responses in synucleinopathies. J. Biol Chem. 285, 9262-9272.

Lee, S.I., Lee, B.R., Hwang, Y.S., Lee, H.C., Rengaraj, D., Song, G., Park, T.S., and Han, J.Y. (2011). MicroRNA-mediated posttranscriptional regulation is required for maintaining undifferentiated properties of blastoderm and primordial germ cells in chickens. Proc. Natl. Acad Sci. USA 108, 10426-10431.
Lewis, B.P., Burge, C.B., and Bartel, D.P. (2005). Conserved seed pairing, often flanked by adenosines, indicates that thousands of human genes are microRNA targets. Cell 120, 15-20.

Liu, Z., Yang, D., Xie, P., Ren, G., Sun, G., Zeng, X., and Sun, X. (2012). MiR-106b and MiR-15b modulate apoptosis and angiogenesis in myocardial infarction. Cell. Physiol. Biochem. 29 851-862.

Livak, K.J., and Schmittgen, T.D. (2001). Analysis of relative gene expression data using real-time quantitative PCR and the 2(Delta Delta C(T)) method. Methods 25, 402-408

McLaren, A. (1992). Development of primordial germ cells in the mouse. Andrologia 24, 243-247.

McLaren, A. (2000). Germ and somatic cell lineages in the developing gonad. Mol. Cell. Endocrinol. 163, 3-9.

McLaren, A. (2003). Primordial germ cells in the mouse. Dev. Biol. 262, 1-15.

Medeiros, L.A., Dennis, L.M., Gill, M.E., Houbaviy, H., Markoulaki, S., Fu, D., White, A.C., Kirak, O., Sharp, P.A., Page, D.C., et al (2011). Mir-290-295 deficiency in mice results in partially penetrant embryonic lethality and germ cell defects. Proc. Natl. Acad. Sci. USA 108, 14163-14168.

Olive, V., Jiang, I., and He, L. (2010). mir-17-92, a cluster of miRNAs in the midst of the cancer network. Int. J. Biochem. Cell Biol. 42, 1348-1354.

Pal, R., Totey, S., Mamidi, M.K., and Bhat, V.S. (2009). Propensity of human embryonic stem cell lines during early stage of lineage specification controls their terminal differentiation into mature cell types. Exp. Biol. Med. 234, 1230-1243.

Peng, X., Li, Y., Walters, K.A., Rosenzweig, E.R., Lederer, S.L., Aicher, L.D., Proll, S., and Katze, M.G. (2009). Computational identification of hepatitis $C$ virus associated microRNA-mRNA regulatory modules in human livers. BMC Genomics 10, 373.

Saunders, L.R., Sharma, A.D., Tawney, J., Nakagawa, M., Okita, K., Yamanaka, S., Willenbring, H., and Verdin, E. (2010). miRNAs regulate SIRT1 expression during mouse embryonic stem cell differentiation and in adult mouse tissues. Aging 2, 415-431.

Schmid, R., Grellscheid, S.N., Ehrmann, I., Dalgliesh, C., Danilenko, M., Paronetto, M.P., Pedrotti, S., Grellscheid, D., Dixon, R.J., Sette, C., et al. (2013). The splicing landscape is globally reprogrammed during male meiosis. Nucleic Acids Res. 41, 10170-10184.

Sirotkin, A.V., Ovcharenko, D., Grossmann, R., Laukova, M., and Mlyncek, M. (2009). Identification of microRNAs controlling human ovarian cell steroidogenesis via a genome-scale screen. J. Cell. Physiol. 219, 415-420.

Takada, S., Berezikov, E., Choi, Y.L., Yamashita, Y., and Mano, H. (2009). Potential role of miR-29b in modulation of Dnmt3a and Dnmt3b expression in primordial germ cells of female mouse embryos. RNA 15, 1507-1514.

Tang, F. (2010). Small RNAs in mammalian germline: tiny for immortal. Differentiation 79, 141-146.

Tong, M.H., Mitchell, D.A., McGowan, S.D., Evanoff, R., and Griswold, M.D. (2012). Two miRNA clusters, Mir-17-92 (Mirc1) and Mir-106b-25 (Mirc3), are involved in the regulation of spermatogonial differentiation in mice. Biol. Reprod. 86, 72.

Tran, D.H., Satou, K., and Ho, T.B. (2008). Finding microRNA regulatory modules in human genome using rule induction. BMC Bioinform. 9 Suppl 12, S5.

Wang, $X$. (2008). miRDB: a microRNA target prediction and functional annotation database with a wiki interface. RNA 14, 1012-1017.

Watson, C.M., and Tam, P.P. (2001). Cell lineage determination in the mouse. Cell Struct Funct. 26, 123-129.

Xu, F., Gao, Z., Zhang, J., Rivera, C.A., Yin, J., Weng, J., and Ye, J. (2010). Lack of SIRT1 (Mammalian Sirtuin 1) activity leads to liver steatosis in the SIRT1 ${ }^{+/-}$mice: a role of lipid mobilization and inflammation. Endocrinology 151, 2504-2514.

Zhang, Y., Hayashi, Y., Cheng, X., Watanabe, T., Wang, X., Taniguchi, N., and Honke, K. (2005). Testis-specific sulfoglycolipid, seminolipid, is essential for germ cell function in spermatogenesis. Glycobiology 15, 649-654.

Zhou, B., Li, C., Qi, W., Zhang, Y., Zhang, F., Wu, J.X., Hu, Y.N., Wu D.M., Liu, Y., Yan, T.T., et al. (2012). Downregulation of miR181a upregulates sirtuin-1 (SIRT1) and improves hepatic insulin sensitivity. Diabetologia 55, 2032-2043. 